\title{
Sciendo
}

DOI: 10.2478/aucts-2019-0001

ACTA UNIVERSITATIS CIBINIENSIS - TECHNICAL SERIES

Vol. 712019

MULTIMODALITY IMAGING IN ISCHEMIC CARDIOMYOPATHY

\author{
Florina BOLEA (BATAR) \\ Faculty of Medicine, Lucian Blaga University Sibiu, Romania, florinabolea@hotmail.com \\ Gabriela EMINOVICI \\ Faculty of Medicine, Lucian Blaga University Sibiu, Romania \\ Andra-Maria BEBESELEA \\ Faculty of Medicine, Lucian Blaga University Sibiu, Romania, andra.bebeselea@gmail.com \\ Ioan MANITIU \\ Faculty of Medicine, Lucian Blaga University Sibiu, Romania, ioanmanitiu@yahoo.com
}

\begin{abstract}
Ischemic heart disease is the most common heart pathology in medical practice. Proper assessment of the size of myocardium injury and its consequences on the heart function is extremely important both for a correct drug therapy and for interventional approach. Most frequently the left ventricle is affected. The need for accurate evaluation of the impact of left ventricular myocardial damage has led to the development of new imaging techniques and improvement of the existing ones. At the moment the most commonly used imaging method for the assessment of left ventricular function is echocardiography. Cardiac Magnetic Resonance and nuclear tomography are precious imaging techniques as well. They are more expensive but bring valuable information when used in adequate situations.
\end{abstract}

Keywords: magnetic resonance, ultrasonography, scintigraphy

\section{Left Ventricular modifications in ischemic heart disease}

Myocardial ischemia decreases ventricular performance at a coronary flow reduction of over $50 \%$, being directly related with the severity of ischemia. Myocardial segments are affected in correlation with the affected arterial segment interested by ischemia. The systolic function of the left ventricle can be affected in what concerns segmental contraction or global contractility. Diastolic dysfunction is present whenever the systolic dysfunction exists and, in most situations, it precedes systolic dysfunction. The depression of the systolic function can have variable intensity accordingly to de decrease of coronary flow: hypokinesia (which expresses a contractility reduction in certain areas - when there is a decrease of coronary flow of over 70-80\%), akinesia (which is the total loss of contractility in a myocardial segment - occurring at a coronary flow lower than $80-85 \%$ ) and dyskinesia (which represents the movement of the myocardium in the opposite direction during systole - it appears at a reduction of coronary flow of over 95\%)

In patients with ischemic cardiomyopathy it is extremely is important to recognize the segments of hibernating myocardium and of the areas of stunning regions because these regions have potentially are reversible ischemia, whereas necrotic tissue and fibrosis have no potential of recovering tissue and therefor contractility. The term of stunning myocardium refers to myocardial mass which could become hypofunctional or can completely lose function after a severe transient ischemia, an area that remains dysfunctional for hours or days after the resolution of ischemia and has a potential to regain normal function spontaneously in about one week in the absence of recurrent ischemia [1]. The hibernating myocardium is defined as dysfunctional myocardium that improves contractility function after revascularization [2].

As a consequence of myocardial infarction ventricular remodeling occurs. It includes both the infarcted and non-infarcted areas and involves changes in the size of the left ventricle, its shape and the wall thickness that will affect the function of the LV. Ventricular remodeling involves two processes: expansion of the infarcted area (growth and thinning of the wall without additional myocardial necrosis) 
and ventricle dilatation. The apex is the segment with the thinnest wall, and it is the most sensitive and predisposed to this phenomenon.

Ventricular dilation is the second component of ischemic ventricular remodeling. This expansion of the non-infarcted area of the LV is a compensatory mechanism which aims to maintain the left ventricular ejection volume. If a sufficient amount of myocardium suffers consequently to an ischemic event, the left ventricular pump function depreciates, the filling pressure and the stroke volume decreases and the endsystolic volume increases, leading to signs of heart failure. Another cause of heart failure is ischemic mitral regurgitation. Ischemic papillary muscle rupture produces acute severe mitral regurgitation. The causes of chronic mitral valve regurgitation are ischemia of papillary muscle which leads to dysfunction, fibrosis and ventricular remodeling [3].

\section{Echocardiography}

Echocardiography uses 2D,3D and Doppler techniques to directly visualize the heart and vessels [4].

Echocardiography is a crucial investigation in diagnosis and follow up of patients with heart failure. This exploration brings data regarding cardiac geometry, systolic and diastolic function of the left ventricle, complications, it can assess kinetic disorders. Besides vital information that echocardiography gives about the functioning of the left ventricle, it describes changes in the morphology of the left ventricle in heart failure due to ischemic heart disease: dilatation of the left ventricle, mitral valve regurgitation, distortion of the LV. The first change that occurs at the level of ischemic muscle is alteration in the thickening systolic segmentation that can be detected by M Mode and 2D ultrasonography. Ventricular aneurysm is detected by a dilatation of myocardial segments that join the dyskinetic movement, reducing myocardial wall thickness at this level and giving the scar appearance [5].

In severe chronic ischemia, in large aneurysms that occupy over half of the ventricular cavity, kinetic disorders can become global, creating the appearance of ischemic dilated cardiomyopathy. To quantify the severity of ischemic myocardial mass for each segment of the 17's proposed by the American Society of Cardiology, it is assigned a score ranging from 1 to 5 (1- meaning normal contraction, 2-hipokinsia, 3akinesia, 4-5, dyskinesia and LV aneurysm). The score obtained is directly proportional to the severity of ischemia [6]. Ultrasound evaluation of chronic heart failure of any cause is obtained by the quantification of the left ventricle ejection fraction (LVEF). LVEF can be calculated by several methods. Linear measurements of the left ventricle in systole and in diastole using M Mode define segmental ejection fraction and shortening fraction of LV. This method has limitations in the evaluation of ischemic heart failure due to the particular shape of the LV and the fact that the LV function may vary in different regions and therefor it can either overestimate or underestimate the LVEF. The Simpson method using 2D Ultrasound is the most common method of assessment of LVEF. However, if ventricular asynchronism exists, the accuracy of the method decreases [7].

Doppler echocardiography is the mostly utilized imaging technique in the evaluation of ventricular function due to the multitude of information that it provides, accuracy and the possibility of follow up without risks. The parameters that are used are: left ventricular ejection tract velocity time integral - VTI LVOT (which assesses global systolic function-independent of the body surface), the rate of increase of LV pressure tissue systolic velocity, (evaluates segmental function), isovolumetric acceleration (brings information about global contractility function). Myocardial deformation (changes in the length of the segment correlated to its initial length) is altered in ischemic heart failure because of decreased systolic deformation (of the infarcted area) and postsystolic deformation of the LV myocardium [8]. The use of myocardial velocity and myocardial deformation allows an interpretation of regional myocardial dysfunction of finesse and an early detection of myocardial function alteration.

\section{MRI in ischemic heart failure}

MRI is used to describe the structures and functions of mycardial tissue and valves. MR devices use magnetic fields and radio waves [9].

Cardiac MRI has the classic indications for the evaluation of the anatomy of the cardiovascular structures and has recently acquired a central role for the characterization of myocardium, with applications in the study of myocardial viability after an ischemic event or the study of myocardial infiltrative diseases. 
The magnetic resonance imaging technique is the gold standard for quantifying ventricular volumes and masses due to its three-dimensional nature that is not based on geometric simulation [10]. Measurement accuracy in the determination of the volume of the left ventricle have been proven by numerous studies in vivo and in vitro

CMR is a valuable tool in evaluating regional contractile function. Images are acquired by sequences of cine-MRI. Quantification of kinetics and wall thickness using conventional techniques is possible but is not used on a large scale. A new technique developed to increase performance of software and image resolution can use certain components of the myocardium to assess myocardial deformation [11] .

The presence of a thinned wall suggests a history of myocardial infarction. Myocardial infarction complications such as thrombosis, an aneurysm, intramural rupture of the interventricular septum or of the papillary muscles are easy to recognize CMR. Changes in the LV ejection function and volume in the same individual due to the progression of the disease or the therapeutic intervention effect can be followed using CMR. In recent years, CMR is a very good method for examining tissue viability and ischemia. In the chronic phase of ischemic heart disease CMR may become the tool used for stratification and therapeutic intervention guidance [14]. The use of late enhancement CMR and stress CMR using dobutamine is recommended especially due to increased risk of recurrence of ischemic events. Gadolinium distribution volume is much higher in scar tissue that in the viable myocardium. When using dobutamine stress testing, the hibernating myocardium that can benefit from revascularization can be identified. For identifying the viable myocardium late gadolinium enhancement (LGE) is used (10-30 minutes after the injection of gadolinium). The identification of viable myocardium and scar tissue throughout the use of CRM has been demonstrated to be a predictive factor for the esponse to cardiac resynchronize therapy [12]. Cardiac magnetic resonance is a precise and reproducible method, useful in the evaluation of the function, anatomy and ischemic areas of left ventricular myocardial in ischemic heart failure.

\section{Myocardial scintigraphy}

Myocardial scintigraphy is an important tool for clinical assessment of patients with probable ischemic heart disease, having in mind its high diagnostic precision [13].

- Single-photon emission computed tomography (SPECT)

Single-photon emission computed tomography is a nuclear medicine tomographic imaging technique using gamma rays. This information is typically presented as cross-sectional slices of the patient's structures but can be freely reformatted or manipulated as required.

A radionuclide is injected into the patient after vein cannulation, and a special radioisotope is bind to the specific ligand, that is seen with the gamma camera. This method evaluates the adsorption technetium-99 or thallium-201 in normal cardiac cells [14]. The technique is available in many centers, with some specific limitations [15].

- Positron emission tomography (PET)

Positron-emission tomography is one of the nuclear techniques which is used for evaluation of cell metabolism. The technique measures gamma rays derived from fluorine-18. [16].

Positron emission tomography is used more and more often in order to see if there is hibernating myocardium, fibrosis of normal tissue [17]. PET has a reasonable sensitivity, but still limited specificity [18]. While the quality of the images is very good, still the technique is quite expensive [19].

Stress tests combined with nuclear techniques are novel tests which help find the specific coronary vessel responible for the damaged tissue. Also, it helps with the identification of low risk patients [20]. As in the case of SPECT, patients that had a normal PET, had $0.4 \%$ annual rates of cardiac events [21].

\section{References}

1. Eli R., Marban E.: Molecular and cellular mechanisms of myocardial stunning. Physiol Rev 1999; 79:609

2. Canty J.M., Fallavollita J.A. Hibernating myocardium. J Nucl Cardiol 2005 ; 12: 104 
3. Borger M.A., Alam A., Murphy P.M., (2006 )et al: Chronic ischemic mitral regurgitation: Repair,replace or rethink?. Ann Thorac Surg; 81:1153.

4. Cleve, Jayne; McCulloch, Marti L. "Conducting a Cardiac Ultrasound Examination", Echocardiography, Springer International Publishing, (2018), pp. 33-42

5. Zdrenghea D, Andronache M. (1998) Diagnosticul cardiopatiei ischemice - Ecocardiografia, in Zdrenghea D, Olinic N. :Cardiopatia ischemica,Cluj Napoca Ed Clusium, 151-16010.

6. Feigenbaum W.F., Amstrong T Feigenbaum's Echocardiography, sixth ed, Ed. Lippincott,Williams and Wilkins, (2005) 138-146

7. Popescu B., Beladan C., Ticulescu R. (2011) Evaluarea Doppler a functiei ventriculului stang in Popescu B si Ginghina C : Ecocardiografia Doppler, Bucuresti, Ed Medicala, 69-81

8. Smith-Bindman R, Miglioretti DL, Johnson E, Lee C, Feigelson HS, Flynn M. Use of diagnostic imaging studies and associated radiation exposure for patients enrolled in large integrated health care systems, 1996-2010. JAMA. 2012 Jun 13;307(22):2400-9

9. Bellenger, Burgess, Ray M SG, et al (2000) Comparison of left ventricular ejection fraction and volumes in heart failure by two-dimensional radionuclide ventriculography, echocardiography and cardiovascular magnetic resonance: Are they interchangeable? Eur Heart ; 21: 138717.

10. My VM, Axel L. Advances in MRI tagging techniques for determining regional myocardialstrain. Cardiol Rep; 2006: 8:-53.

11. Schwitter, J., Array of: cardiac ischemia and assessmant of viability: of cardiovascular magnetic resonance. Eur Heart J 32;2011: 799-81

12. Rutz A.K., Manka R., Kozerke S., Roas S., Boesiger P., Schwitter J. Left ventricular dyssynchrony in patients with left bundle branch block and patients after myocardial infarction: integration of mechanics and viability by cardiac magnetic resonance. Eur Heart J, (2009) 30:2117-2127

13. Smanio PE, Buchpiguel CA. Timerman A, Bertolami MC, Ferreira FM. Manual de cardiologia. São Paulo: Atheneu; 2012. Medicina Nuclear: Princípios gerais, indicações e interpretação; pp. 781-789

14. Bogaert J, Gheysens O, Dymarkowski S, Goetschalckx K. Comprehensive evaluation of hibernating myocardium: use of noninvasive imaging. J Thorac Imaging. 2014; 29(3):134-46

15. Schuster A, Morton G, Chiribiri A, Perera D. Imaging in the Management of Ischemic Cardiomyopathy. JACC , 2012;59 (4):359-70

16. Bailey, D.L; D.W. Townsend; P.E. Valk; M.N. Maisey (2005). Positron-Emission Tomography: Basic Sciences. Secaucus, NJ: Springer-Verlag

17. Schuster A, Morton G, Chiribiri A, Perera D. Imaging in the Management of Ischemic Cardiomyopathy. JACC , 2012;59 (4):359-70

18. Schinkel AF, Bax JJ, Poldermans D, Elhendy A, Ferrari R, Rahimtoola SH. Hibernating myocardium: diagnosis and patient outcomes. Curr Probl Cardiol. 2007; 32(7):375-410

19. Lima GL, Camara de Caralho FP. Ischemic left ventricle systolic dysfunction: An evidence-based approach in diagnostic tools and therapeutics. Rev Assoc Med BraS 2017; 63(9):793-800

20. Berman D.S., Hachamovitch R., Shaw L.J., et al. Roles of nuclear cardiology, cardiac computed tomography, and cardiac magnetic resonance: noninvasive risk stratification and a conceptual framework for the selection of noninvasive imaging tests in patients with known or suspected coronary artery disease. Journal of Nuclear Medicine, 47 (2006), pp. 1107-1118

21. Yoshinaga K., Chow B.J., Williams K., et al. What is the prognostic value of myocardial perfusion imaging using rubidium-82 positron emission tomography? Journal of the American College of Cardiology, 48 (2006), pp. 1029-1039 